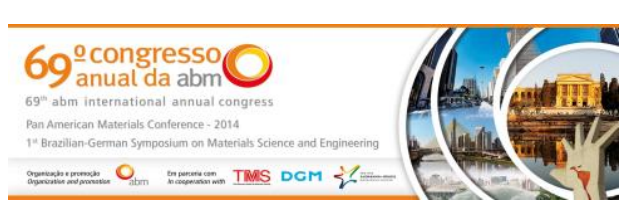

Tema: Iniciação Científico-tecnológica

\title{
CORREÇÃO DA RIGIDEZ À FLEXÃO EM ENSAIOS DE PLACAS ÓSSEAS METÁLICAS*
}

\section{Resumo}

Agnes Ágata Fróes Martins ${ }^{1}$ Afonso Reguly ${ }^{2}$

Bill Paiva dos Santos ${ }^{3}$

Cínthia Gabriely Zimmer ${ }^{4}$

Claudia Lisiane Fanezi da Rocha ${ }^{5}$

Daniel Antonio Kapper Fabricio ${ }^{6}$ Vagner Machado Costa ${ }^{7}$

Placas ósseas metálicas são componentes utilizados para correção ou cura de fraturas ósseas. Em se tratando de implantes ortopédicos, é fundamental conhecer o comportamento dos materiais quando submetidos a esforços mecânicos. Para isto ensaios são realizados baseados em normas técnicas, que no caso específico de placas ósseas, são utilizadas as normas ASTM F382 e ABNT NBR 15672-2. Na execução do ensaio para determinação da rigidez à flexão, de uma placa óssea, pode existir o efeito da flexibilidade do dispositivo de ensaio. Para verificar a magnitude desse efeito, foram realizados três ensaios de flexão estático e um ensaio de flexibilidade do dispositivo de ensaio, onde o ensaio foi interrompido ao final da fase elástica. As propriedades mecânicas da placa foram avaliadas, aplicando a correção da flexibilidade do dispositivo. Os resultados mostraram que para os níveis de carregamento alcançados, as cargas de prova apresentaram pouca influência na flexibilidade do dispositivo, e consequentemente pouca influência na rigidez após os cálculos de correção.

Palavras-chave: Rigidez à flexão; Correção; ASTM F382; ABNT NBR 15676-2.

\section{Abstract}

\section{CORRECTION OF FLEXURAL RIGIDITY ON BONE PLATES TESTING}

Plate bones are components used to fix or heal bone fractures. Talking about orthopedic implants, it is essential to understand the material behavior when subjected to mechanical stresses. Mechanical tests are performed based on technical standards, where for bone plates, ASTM F382 and ABNT NBR 15672-2 are used. The effect of the flexibility of the test device can be affect the test result to determine the flexural rigidity of a bone plate. To check the magnitude of this effect, static bending tests and flexibility test of device were executed. The mechanical properties of the plate were evaluated by applying the correction device flexibility. The results showed that the flexibility of the device had little influence on the flexural rigidity.

Keywords: Bend testing; Compensation; ASTM F1264-03; ABNT NBR 15668-2.

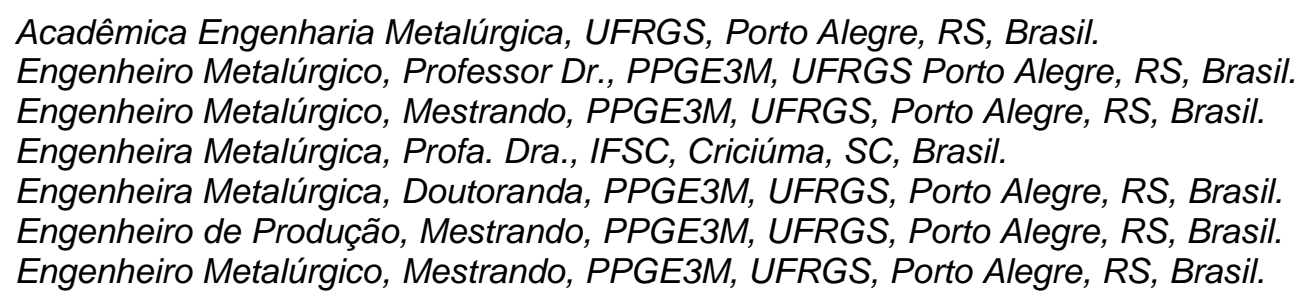

\footnotetext{
* Contribuição técnica ao 69ำ Congresso Anual da ABM - Internacional e ao 14ํㅡㄹ ENEMET - Encontro Nacional de Estudantes de Engenharia Metalúrgica, de Materiais e de Minas, 21 a 25 de julho de 2014, São Paulo, SP, Brasil.
} 


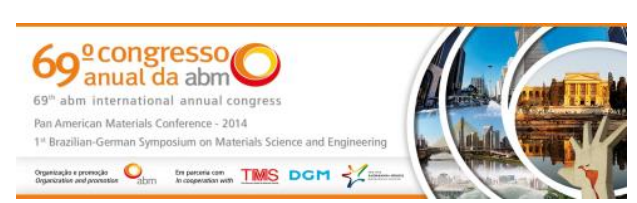

\section{INTRODUÇÃO}

Placas Ósseas são componentes estruturais, também denominadas próteses ortopédicas, as quais são geralmente montadas no sistema esquelético do corpo humano para ajudar a curar, corrigir e restaurar as deformidades ou funções perdidas da estrutura original [1]. A imagem da Figura 1 mostra, de forma esquemática, a utilização desse componente.

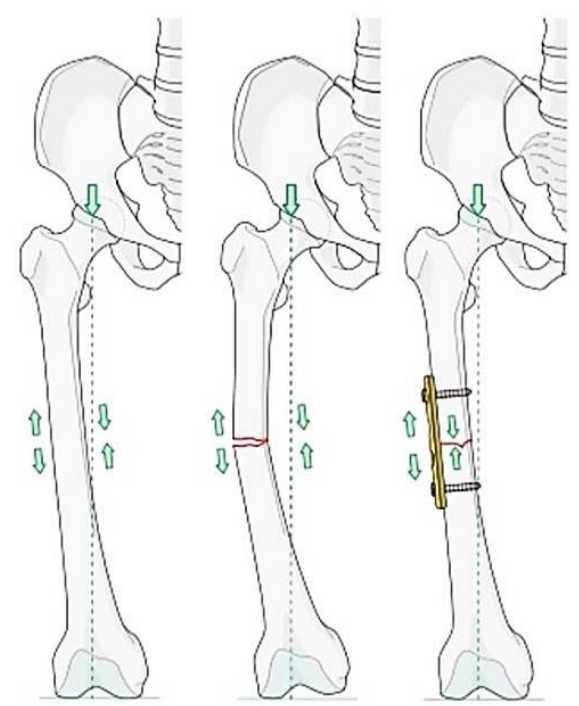

Figura 1. (a) Imagem esquemática mostrando um caso de fratura do fêmur e a utilização de uma placa óssea. Fonte: [2] modificado.

Para que estes componentes cumpram sua função, que é permitir o retorno precoce da função do membro lesionado, ensaios metalúrgicos são realizados para averiguar a qualidade do material; e ensaios mecânicos são realizados para garantir a funcionalidade do componente. Os ensaios mecânicos reproduzem as cargas mais comuns de carregamentos encontrados in vivo, que para o caso de placas ósseas utilizadas no fêmur, é o carregamento é fletivo. Estes ensaios são realizados baseados em normas técnicas, as quais para placas ósseas são as da série ABNT NBR ISO 15676 [3] e ASTM F 382 [4].

O ensaio de flexão estático, um dos ensaios requeridos por norma, será estudado no presente estudo. Neste ensaio recomenda-se uma estimativa de escoamento convencional $(0,2 \%$ de escoamento), obtida em um gráfico: carga versus deslocamento. No gráfico, representa-se a carga total do sistema (duas vezes a carga aplicada em cada suporte) e o deslocamento, representado pela deflexão nos pontos de carga em relação aos suportes na direção y (ou direção vertical). Contudo algumas fontes de erro durante o ensaio devem ser consideradas nos resultados.

Fontes típicas de erro incluem: medições de vão, flexibilidade do dispositivo do suporte, flexibilidade dos acessórios e por fim, a carga de cisalhamento produzida nos pontos de carregamento e de suporte, proporcional à flexão produzida [5].

Considerando que a carga máxima do ensaio corresponde ao escoamento estabelecido, é necessário realizar a devida correção da flexibilidade do dispositivo a partir do cálculo do coeficiente de rigidez da placa óssea.

O coeficiente de flexibilidade é a capacidade que uma estrutura tem de se deformar por unidade de força, em cada um de seus pontos de aplicação. Já o coeficiente de

\footnotetext{
* Contribuição técnica ao 69ำ Congresso Anual da ABM - Internacional e ao 14ํㅡㄹ ENEMET - Encontro Nacional de Estudantes de Engenharia Metalúrgica, de Materiais e de Minas, 21 a 25 de julho de 2014, São Paulo, SP, Brasil.
} 


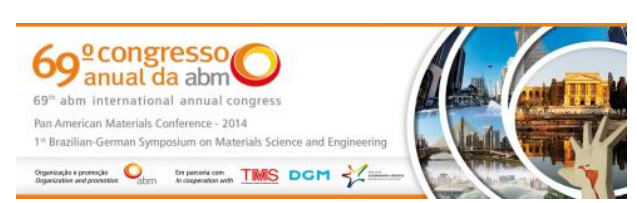

rigidez é definido como a força necessária para produzir uma deformação unitária [6], ou seja, no caso dos metais a deformação plástica.

Nesse sentido este trabalho buscou quantificar os valores de uma das fontes de erro do ensaio de flexão estático, que é a flexibilidade do dispositivo do suporte.

\section{MATERIAIS E MÉTODOS}

A Figura 2 mostra o componente, alvo deste estudo, denominado placas de osteossíntese do tipo DCP (Dynamic Compression Plate), utilizadas para fraturas do fêmur. As placas foram produzidas a partir de aço inoxidável austenítico.

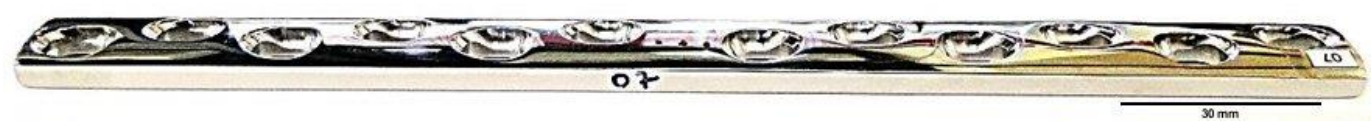

Figura 2. Placa óssea do tipo DCP.

Com intuito de verificar a composição química da placa óssea metálica, foram realizadas três queimas em um espectrômetro de emissão óptica marca Quantron Magelan, sempre observando a repetibilidade dos resultados, para então ser extraída a média. A partir da média dos resultados, eles foram comparados à faixa de composição convencionada pelas normas ABNT NBR ISO 5832-1 [7] e ASTM F 138 [8].

O ensaio de flexão estático foi realizado em uma máquina de ensaios ServoHidráulica (marca MTS, modelo 810 Material Test System, equipada com uma célula de carga de $100 \mathrm{KN}$ ), conforme recomendações da norma NBR ISO 15676-2 [3] e ASTM F 382 [4]. Estas normas descrevem os ensaios de flexão em quatro pontos, que consiste em determinar as propriedades estruturais intrínsecas de placas metálicas.

Três placas ósseas foram ensaiadas à temperatura ambiente $\left(23^{\circ} \mathrm{C} \pm 5^{\circ} \mathrm{C}\right)$, com taxa de deslocamento de $1 \mathrm{~mm} /$ minuto. O dispositivo para o ensaio consistiu em dois roletes de carregamento acoplados ao travessão móvel da máquina de ensaio, e posicionados de modo que cada rolete estivesse entre dois furos da placa. Outros dois roletes de suporte presos à base da máquina e posicionados simetricamente a uma distância de dois furos da placa em relação aos roletes atuadores.

Os roletes apresentavam $15 \mathrm{~mm}$ de diâmetro, os quais permitem minimizar o atrito entre a placa e os cilindros transmissores da carga. A distância entre os roletes de carregamento "a" e a distância entre os roletes de suporte e de carregamento adjacentes, chamado vão de carregamento "h", apresentaram 38mm. A Figura3(a) ilustra o sistema utilizado para este ensaio.

Para o ensaio de flexibilidade do dispositivo, foram utilizados os mesmos dispositivos do ensaio de flexão estático, porém os roletes de suporte foram alinhados aos roletes de carregamento. Foi utilizada taxa de deslocamento em compressão de $0,1 \mathrm{~mm} / \mathrm{s}$. Este ensaio foi baseado nas recomendações da norma ABNT NBR 15668-2 [9] e ASTM F1264 [10]. A Figura3(b) ilustra o sistema utilizado para este ensaio.

A flexibilidade do dispositivo foi calculada através da determinação do valor inverso da inclinação da curva carga-deslocamento na região elástica, expressa em milímetros por Newton $(\mathrm{mm} / \mathrm{N})$.

\footnotetext{
* Contribuição técnica ao $69^{\circ}$ Congresso Anual da ABM - Internacional e ao 14ํㅡㄹ ENEMET - Encontro Nacional de Estudantes de Engenharia Metalúrgica, de Materiais e de Minas, 21 a 25 de julho de 2014, São Paulo, SP, Brasil.
} 


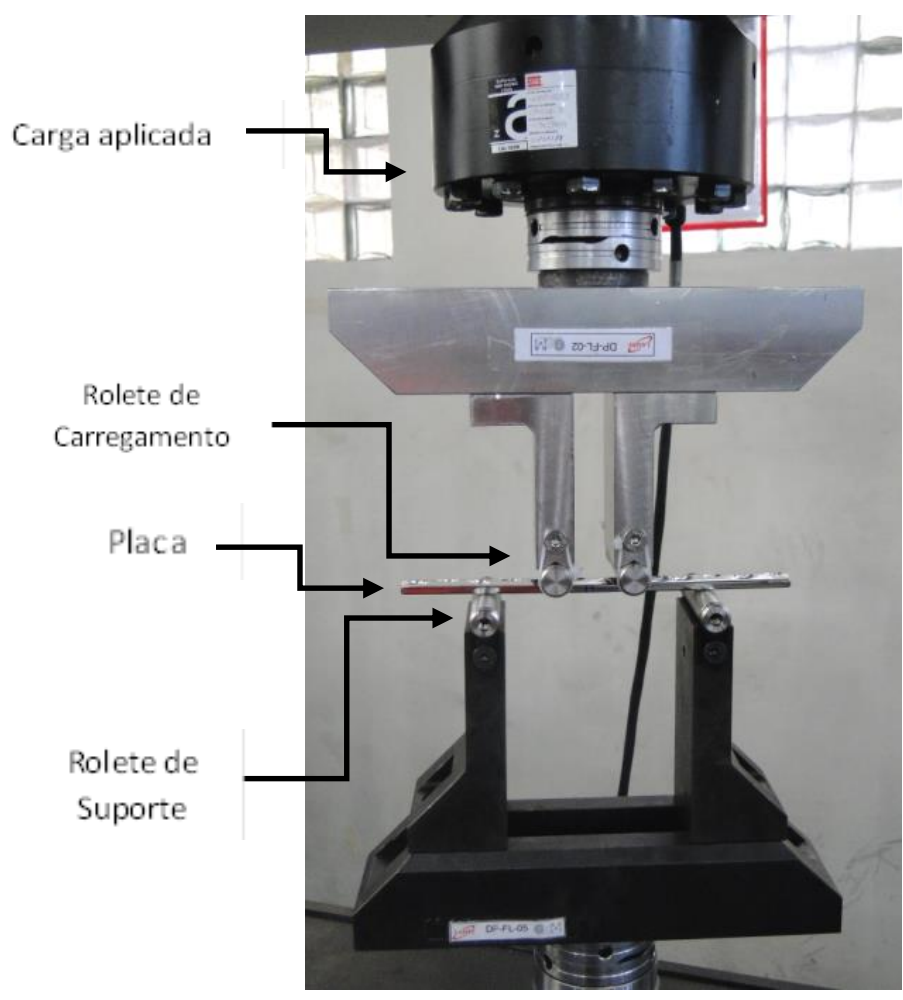

(a)

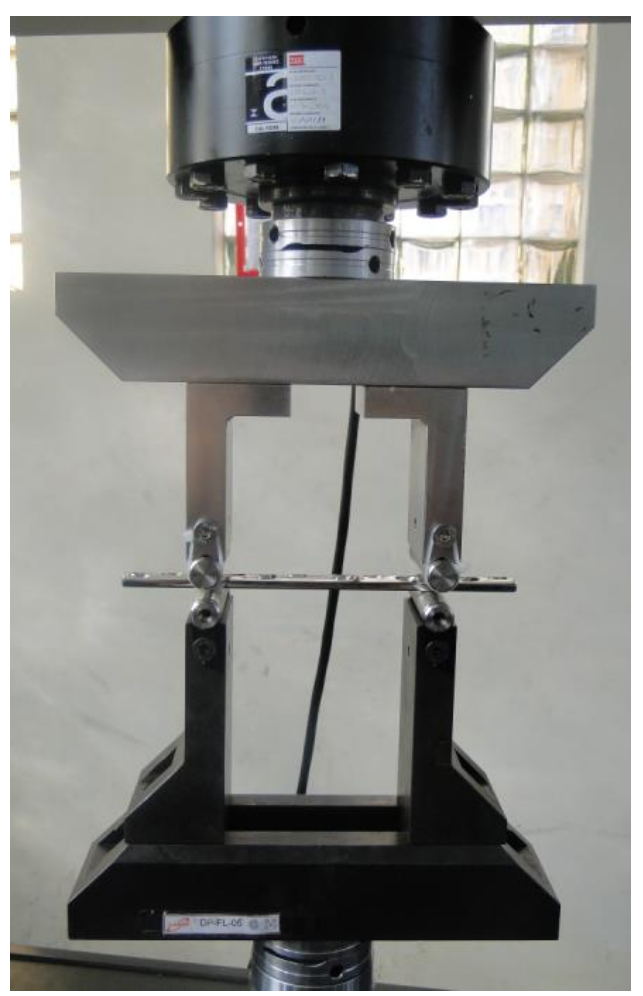

(b)

Figura 3. (a) Arranjo do ensaio de flexão estático. (b) Arranjo do ensaio de flexibilidade do dispositivo.

\section{RESULTADOS E DISCUSSÃO}

A Tabela 1 mostra a composição química da placa óssea metálica em comparação às especificações das normas que conferem a conformidade desses componentes. Os resultados mostram que a composição química está dentro das faixas especificadas, sendo esta composição característica de aço inoxidável austenítico.

Tabela 1. Composição química.

\begin{tabular}{c|c|c|c|c|c|c|c|c|c|c|c}
\hline \multicolumn{10}{c}{ COMPOSIÇÃO QUÍMICA (\% em massa) } \\
\hline $\begin{array}{c}\text { Elemento } \\
\text { Químico }\end{array}$ & $\mathrm{C}$ & $\mathrm{Si}$ & $\mathrm{Mn}$ & $\mathrm{P}$ & $\mathrm{N}$ & $\mathrm{S}$ & $\mathrm{Cr}$ & $\mathrm{Ni}$ & $\mathrm{Mo}$ & $\mathrm{Cu}$ & $\mathrm{Fe}$ \\
\hline $\begin{array}{c}\text { Placa } \\
\text { analisada }\end{array}$ & 0,024 & 0,322 & 1,875 & 0,013 & 0,300 & 0,0017 & 17,14 & 14,64 & 2,640 & 0,059 & Balanço \\
\hline $\begin{array}{c}\text { ABNT NBR } \\
\text { ISO 5832-1 }\end{array}$ & $\begin{array}{c}0,030 \\
\text { máx }\end{array}$ & $\begin{array}{c}1,0 \\
\text { máx }\end{array}$ & $\begin{array}{c}2,0 \\
\text { máx }\end{array}$ & $\begin{array}{c}0,025 \\
\text { máx. }\end{array}$ & $\begin{array}{c}0,10 \\
\text { máx. }\end{array}$ & $\begin{array}{c}0,010 \\
\text { máx. }\end{array}$ & $\begin{array}{c}17,0- \\
19,0\end{array}$ & $\begin{array}{c}13,0- \\
15,0\end{array}$ & $\begin{array}{c}2,25- \\
3,0\end{array}$ & $\begin{array}{c}0,50 \\
\text { máx. }\end{array}$ & Balanço \\
\hline $\begin{array}{c}\text { ASTM } \\
\text { F138-08 }\end{array}$ & $\begin{array}{c}0,030 \\
\text { máx }\end{array}$ & $\begin{array}{c}0,75 \\
\text { máx }\end{array}$ & $\begin{array}{c}2,0 \\
\text { máx }\end{array}$ & $\begin{array}{c}0,025 \\
\text { máx. }\end{array}$ & $\begin{array}{c}0,10 \\
\text { máx. }\end{array}$ & $\begin{array}{c}0,010 \\
\text { máx. }\end{array}$ & $\begin{array}{c}17,0- \\
19,0\end{array}$ & $\begin{array}{c}13,0- \\
15,0\end{array}$ & $\begin{array}{c}2,25- \\
3,0\end{array}$ & $\begin{array}{c}0,50 \\
\text { máx }\end{array}$ & Balanço \\
\hline
\end{tabular}

A Figura 4 apresenta as curvas Carga-versus Deslocamento, resultante dos ensaios de flexão estático. A partir das curvas obtêm-se os valores necessários para calcular a rigidez à flexão da placa. Para calcular a rigidez à flexão utilizou-se a equação 1.

Rigidez à Flexão $=\frac{F_{A}}{D_{A}} \quad$ Equação 1

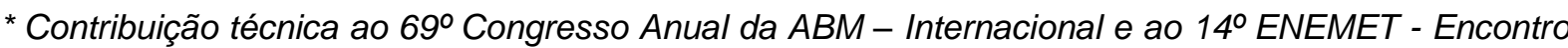
Nacional de Estudantes de Engenharia Metalúrgica, de Materiais e de Minas, 21 a 25 de julho de 2014, São Paulo, SP, Brasil.
} 
Onde:

$F_{A}=$ Força medida no ponto $A$

$D_{A}=$ Deslocamento medido no ponto $A$

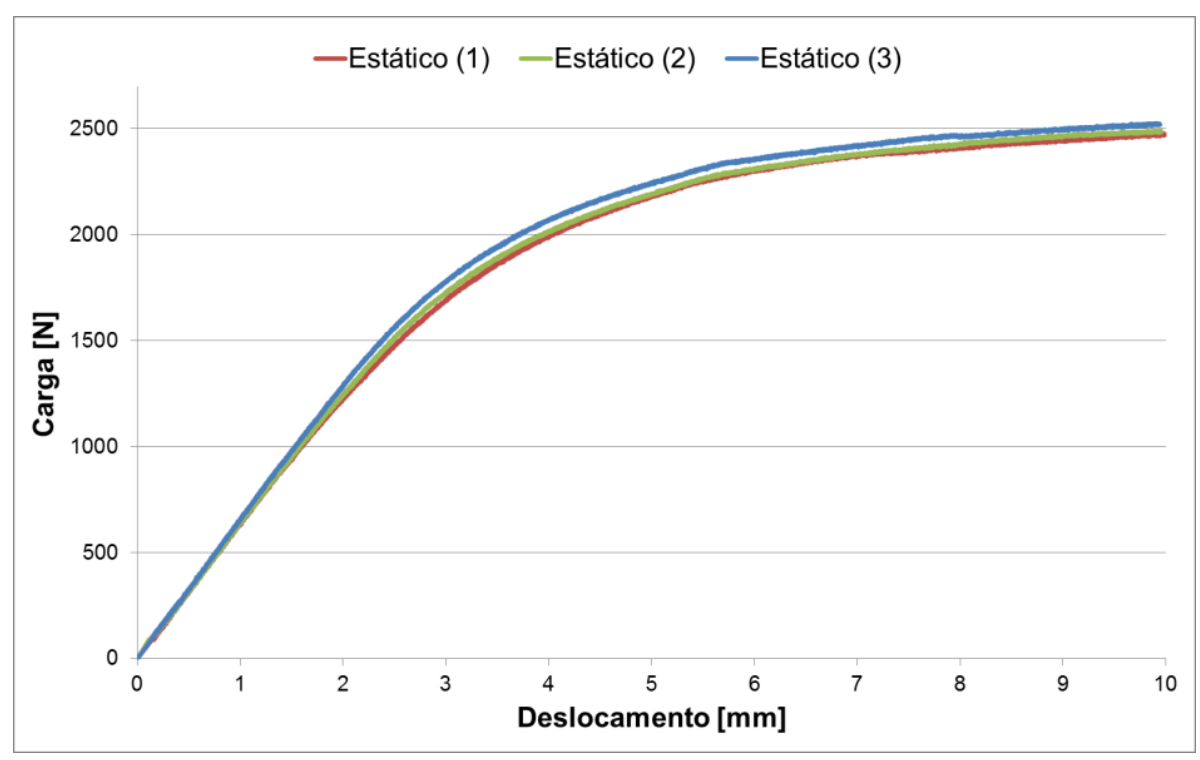

Figura 4. Curvas Carga versus Deslocamento dos ensaios de flexão estático.

A Tabela 2 apresenta os valores das cargas de prova e a rigidez à flexão calculada para cada placa ensaiada.

Tabela 2. Resultados do ensaio de Flexão estático

\begin{tabular}{c|c|c}
\hline Placa & $\begin{array}{c}\text { Cargas de Prova } \\
{[\mathrm{N}]}\end{array}$ & $\begin{array}{c}\text { Rigidez à Flexão } \\
{[\mathrm{N} / \mathrm{mm}]}\end{array}$ \\
\hline 01 & 1328,09 & 636 \\
\hline 02 & 1430,46 & 655 \\
\hline 03 & 1240,22 & 631 \\
\hline Média & 1332,92 & 640,66 \\
\hline
\end{tabular}

A Figura 5 apresenta a curva Carga versus Deslocamento, resultante do ensaio de flexibilidade do dispositivo. A Tabela 3 apresenta os valores da carga máxima e o respectivo deslocamento, obtidos no ensaio. Os valores obtidos neste ensaio serão utilizados na correção do cálculo da rigidez à flexão da placa.

\footnotetext{
* Contribuição técnica ao 69ำ Congresso Anual da ABM - Internacional e ao 14ํㅡㄹ ENEMET - Encontro Nacional de Estudantes de Engenharia Metalúrgica, de Materiais e de Minas, 21 a 25 de julho de 2014, São Paulo, SP, Brasil.
} 

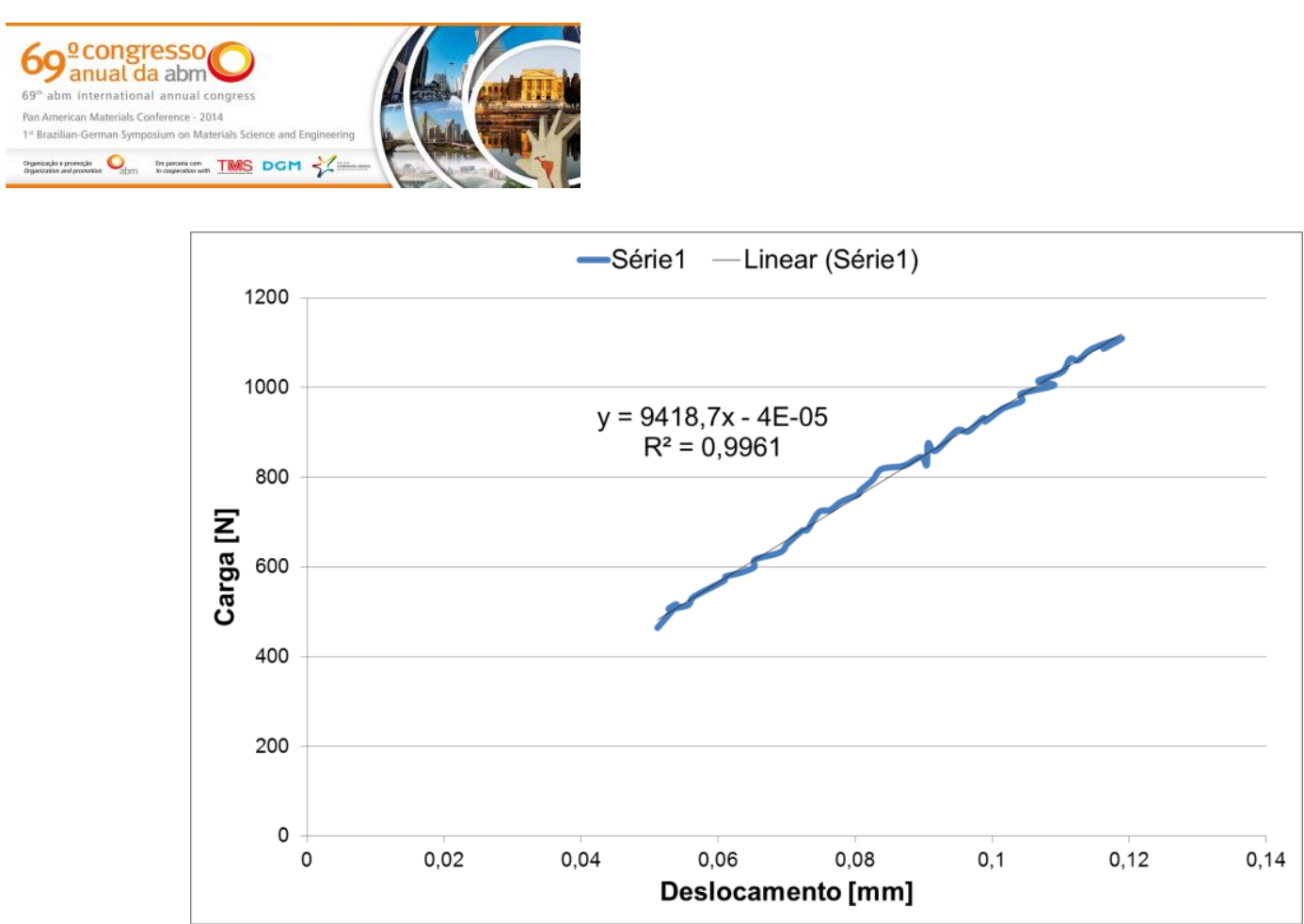

Figura 5. Curva Carga versus Deslocamento do ensaio de flexibilidade do dispositivo.

Tabela 3. Resultados da Flexibilidade do dispositivo de ensaio.

\begin{tabular}{c|c|c}
\hline Placa & $\begin{array}{c}\text { Carga Máxima no } \\
\text { Limite Elástico [N] }\end{array}$ & $\begin{array}{c}\text { Deslocamento Final no } \\
\text { Limite Elástico [mm] }\end{array}$ \\
\hline 01 & 1086,28 & 0,12 \\
\hline
\end{tabular}

A Tabela 4 apresenta as cargas de prova dos ensaios estáticos e as cargas de prova corrigidas, as quais foram determinadas após compensação da flexibilidade do dispositivo.

Tabela 4. Resultados Flexibilidade estrutura/dispositivo

\begin{tabular}{c|c|c}
\hline $\mathrm{CP}$ & $\begin{array}{c}\text { Cargas de Prova } \\
{[\mathrm{N}]}\end{array}$ & $\begin{array}{c}\text { Cargas de Prova } \\
\text { Corrigidas [N] }\end{array}$ \\
\hline 01 & 1328,09 & 1213,61 \\
\hline 02 & 1430,46 & 1416,97 \\
\hline 03 & 1240,22 & 1243,43 \\
\hline Média & 1332,92 & 1291,34 \\
\hline
\end{tabular}

A Tabela 5 apresenta cargas de prova corrigidas e a rigidez à flexão real, ou seja com as devidas correções. As cargas de prova corrigidas foram determinadas após compensação da flexibilidade do dispositivo.

Tabela 5. Resultados após correção.

\begin{tabular}{c|c|c}
\hline Placa & $\begin{array}{c}\text { Cargas de Prova } \\
\text { Corrigidas [N] }\end{array}$ & $\begin{array}{c}\text { Rigidez à Flexão } \\
\text { Corrigidas [N/mm ] }\end{array}$ \\
\hline 01 & 1213,61 & 581 \\
\hline 02 & 1416,97 & 649 \\
\hline 03 & 1243,43 & 632 \\
\hline Média & 1291,34 & 620 \\
\hline
\end{tabular}

Os resultados mostram que a flexibilidade do dispositivo altera a rigidez à flexão real da placa em torno de $3 \%$.

\footnotetext{
* Contribuição técnica ao 69ำ Congresso Anual da ABM - Internacional e ao 14ํㅡㄹ ENEMET - Encontro Nacional de Estudantes de Engenharia Metalúrgica, de Materiais e de Minas, 21 a 25 de julho de 2014, São Paulo, SP, Brasil.
} 


\section{CONCLUSÃO}

De acordo com os resultados obtidos, pode-se concluir que:

- Com as compensações da flexibilidade do dispositivo, as cargas de prova apresentaram valores pouco mais conservadores;

- Considerando que a máquina utilizada para os ensaios apresenta capacidade de trabalho de $100 \mathrm{kN}$, as cargas de prova alcançadas apresentaram pouca influência da flexibilidade estrutura/dispositivo.

- E por fim o objetivo principal deste trabalho, definir a magnitude da flexibilidade do dispositivo de ensaio sob a rigidez da placa, mostrou que pode haver diferenças entre a rigidez apresentada pelo sistema e a rigidez real em torno de $3 \%$.

\section{REFERÊNCIAS}

1 Pohler OE. (2002). Failures of Metallic Orthopedic Implants. In: Metals Handbook 11 Failure Analysis and Prevention (pp. 2740 - 2790). NY: ASM International.

2 Rüedi TP, Buckley RE, Moran CG. (2007). AO Principles of Fracture Management Volume 1 - Principles. Thieme.

3 ABNT NBR 15676-2. (2009). Implantes para Ortopedia - Placas Ósseas Metálicas. Parte 2: Método de Ensaio de Flexão Estática. Associação Brasileira de Normas Técnicas.

4 ASTM F 382. (1999). Standard Specification and Test Method for Metallic Bone Plates. American Society for Testing and Materials.

5 ABNT NBR 15668-2. Implantes para ortopedia - Dispositivos de fixação intramedular. Parte 2: Método de ensaio para flexão estática em quatro pontos de haste intramedular. Rio de Janeiro: ABNT, 2009.

6 Santos AM. Análise matricial preliminar de estrutura estaticamente indeterminadas por meio do método da flexibilidade: Um estudo de caso envolvendo duas vigas hiperestáticas e a determinação completa dos seus deslocamentos, rotações e coeficientes de flexibilidade. Uniuv em Revista, União da Vitória, 2008; 11: 27-72.

7 ABNT NBR 5832-1. Implantes cirúrgicos - Materiais metálicos . Parte 1: Aço inoxidável conformado. Rio de Janeiro: ABNT, 2008.

8 ASTM F138. Standard Specification for Wrought 18Chromium-14Nickel2.5Molybdenum Stainless Steel Bar and Wire for Surgical Implants (UNS S31673). Pennsylvania: ASTM International, 2013.

9 ABNT NBR 15668-2 Implantes para ortopedia - Dispositivos de fixação intramedular Parte 2: Método de ensaio para flexão estática em quatro pontos de haste intramedular. Associação Brasileira de Normas Técnicas.

10 ASTM F1264-03. Standard Specification and Test Methods for Intramedullary Fixation Devices. ASTM International, 2012.

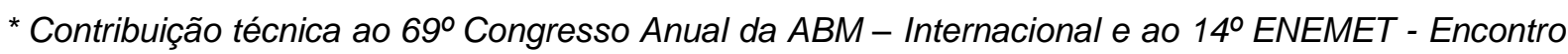
Nacional de Estudantes de Engenharia Metalúrgica, de Materiais e de Minas, 21 a 25 de julho de 2014, São Paulo, SP, Brasil.
} 